\title{
Phytoprotection
}

\section{Détection du Heterobasidion annosum au Québec}

\section{G. Laflamme et R. Blais}

Volume 76, numéro 1, 1995

URI : https://id.erudit.org/iderudit/706083ar

DOI : https://doi.org/10.7202/706083ar

Aller au sommaire du numéro

\section{Éditeur(s)}

Société de protection des plantes du Québec (SPPQ)l

\section{ISSN}

0031-9511 (imprimé)

1710-1603 (numérique)

Découvrir la revue

Citer cet article

Laflamme, G. \& Blais, R. (1995). Détection du Heterobasidion annosum au Québec. Phytoprotection, 76(1), 39-43. https://doi.org/10.7202/706083ar

\section{Résumé de l'article}

Depuis 1955, le champignon Heterobasidion annosum, cause de la maladie du rond, n'était présent, dans l'est du Canada, que dans le sud de l'Ontario. En 1968, le champignon fut identifié plus au nord, près des frontières du Québec. À partir de 1983, un groupe de quatre plantations de pins rouges (Pinus resinosa) ayant déjà été éclaircies a fait l'objet d'un programme de surveillance, ce qui nous a permis de détecter le H. annosum dans la plantation de Mayo en 1989. Cette détection constitue la première mention de la présence du champignon au Québec. En 1993, on a observé la maladie dans une autre plantation du groupe, à Saint-Philippe-d'Argenteuil. Une troisième plantation infectée a aussi été trouvée près de Harrington en 1993, mais elle nefaisait pas partie du groupe sous surveillance. Elle aurait été infectée avant les autres, soit en 1977. La détection du H. annosum au Québec démontre l'urgence d'effectuer des traitements préventifs dans les plantations de pins rouges lors de l'éclaircie et d'éradiquer la maladie dans les trois plantations infectées pendantqu'il en est encore temps. 


\title{
Communication brève/Short Communication
}

\section{Détection du Heterobasidion annosum au Québec}

\author{
Gaston Laflamme et Robert Blais ${ }^{1}$
}

Reçu 1994-07-26; accepté 1995-03-22

Depuis 1955, le champignon Heterobasidion annosum, cause de la maladie du rond, n'était présent, dans l'est du Canada, que dans le sud de l'Ontario. En 1968, le champignon fut identifié plus au nord, près des frontières du Québec. À partir de 1983, un groupe de quatre plantations de pins rouges (Pinus resinosa) ayant déjà été éclaircies a fait l'objet d'un programme de surveillance, ce qui nous a permis de détecter le $H$. annosum dans la plantation de Mayo en 1989. Cette détection constitue la première mention de la présence du champignon au Québec. En 1993, on a observé la maladie dans une autre plantation du groupe, à Saint-Philippe-d'Argenteuil. Une troisième plantation infectée a aussi été trouvée près de Harrington en 1993, mais elle ne faisait pas partie du groupe sous surveillance. Elle aurait été infectée avant les autres, soit en 1977. La détection du $H$. annosum au Québec démontre l'urgence d'effectuer des traitements préventifs dans les plantations de pins rouges lors de l'éclaircie et d'éradiquer la maladie dans les trois plantations infectées pendant qu'il en est encore temps.

Laflamme, G., and R. Blais. 1995. Detection of Heterobasidion annosum in Quebec. PHYTOPROTECTION 76: 39-43.

Since 1955, the fungus Heterobasidion annosum, the causal agent of annosus root rot, was present in Eastern Canada but only in southern Ontario. In 1968, the presence of the fungus was noted further north, near the Quebec border. A group of four already thinned red pine (Pinus resinosa) plantations has been monitored by us since 1983 , leading to the detection of $H$. annosum in the Mayo plantation in 1989. That was the first report of the disease in Quebec. In 1993, we found the disease in another plantation of this group, at Saint-Philipped'Argenteuil. A third infected plantation was also found near Harrington in 1993, but it was not part of the group monitored. It probably became infected before the others, in 1977. The detection of $H$.annosum in Quebec shows the importance of using preventive measures during the thinning of red pine plantations, and of eradicating the disease in the three infected plantations while it is still possible.

Le champignon Heterobasidion annosum (Fr.) Bref. [Fomes annosus (Fr.) Karst.], retrouvé sur tous les continents, provoque une maladie des racines des conifères connue sous le nom de maladie du rond.
En raison de l'importance des dégâts que cause ce champignon, il se classe parmi les agents pathogènes les plus dévastateurs des forêts résineuses (Dimitri 1973).

1. Ressources naturelles Canada, Service canadien des forêts - Québec, 1055, rue du PEPS, C.P. 3800, Sainte-Foy (Québec), Canada G1V 4C7 
Les spores du champignon peuvent voyager sur de longues distances. Rishbeth (1959) a relevé la présence de spores viables, en mer, à plus de $300 \mathrm{~km}$ de la source d'infection la plus proche. Ainsi, après avoir été transportées par le vent, des basidiospores de $H$. annosum vont germer sur la découpe de souches d'arbres fraîchement coupés (Rishbeth 1951). C'est à partir de ces souches colonisées que le champignon va d'abord rayonner en saprophyte à la vitesse d'environ $1 \mathrm{~m}$ année ${ }^{-1}$. Puis, le champignon s'attaquera aux racines vivantes qui sont en contact avec celles de la souche colonisée, provoquant ainsi la mort des arbres sains ou une carie du pied. Par la suite, ces arbres infectés vont eux aussi propager la maladie aux arbres voisins. Ce mode de progression à partir d'un point central crée des zones de mortalité de forme circulaire dans les peuplements forestiers, ce qui a donné son nom à la maladie.

Dans l'est du Canada, c'est en Ontario, sur des pins rouges (Pinus resinosa Ait.), que la maladie a été rapportée pour la première fois (Hord and Quirke 1955). Les deux plantations malades alors détectées avaient été éclaircies environ 10 ans auparavant. Après 1955, la maladie a été identifiée à plusieurs endroits dans le sud de l'Ontario puis, en 1968, Sippell et al. (1968) I'identifièrent plus au nord, à la forêt Larose $\left(45^{\circ} 30^{\prime} \mathrm{N} 75^{\circ} 15^{\prime}\right.$ O) près des frontières du Québec (fig. 1). La maladie affecte plus fréquemment les plantations de pins rouges mais elle a occasionnellement été détectée dans des plantations de pins sylvestres ( $P$. sylvestris L.), de pins gris ( $P$. banksiana Lamb.) et de pins blancs (P. strobus L.) (Sippell et al. 1967).

Au Nouveau-Brunswick, une opération de capture de spores a démontré qu'il existait des spores viables de $H$.annosum dans l'air, même si la maladie n'est pas présente dans cette province (Redfern and Van Sickle 1968). Au Québec, on croyait avoir trouvé le $H$. annosum lors d'une excursion mycologique faite à Duchesnay en 1938 par des membres de la Mycological Society of Arnerica (Mains et al. 1939). Toutefois, un examen ultérieur des spécimens a révélé qu'il ne s'agissait en fait que d'une autre espèce de polypore.

En 1982, le Comité de pathologie forestière du ministère des Ressources naturelles du Québec recommandait aux forestiers de traiter au borax $\left(\mathrm{Na}_{2} \mathrm{~B}_{4} \mathrm{O}_{7}-10\right.$ $\mathrm{H}_{2} \mathrm{O}$ ) les souches de pin rouge résultant des éclaircies, principalement en raison de la présence de la maladie dans la forêt Larose située en Ontario. Dès l'année suivante, une surveillance plus étroite a été effectuée par notre équipe pour détecter les signes d'apparition de cette

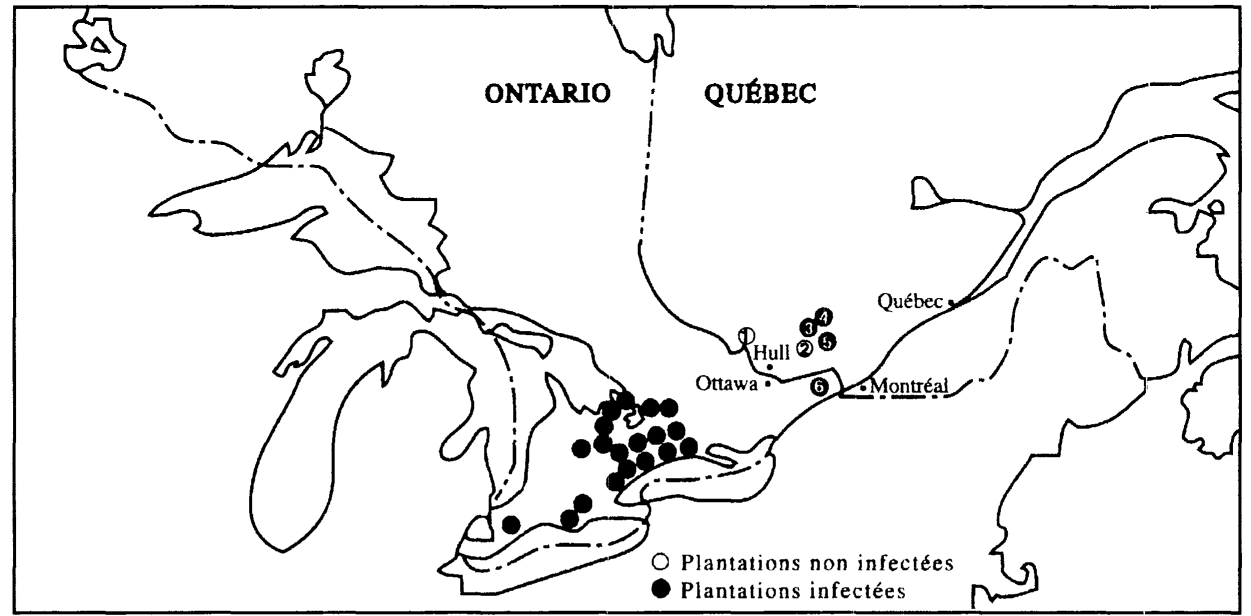

Figure 1. Distribution du $H$. annosum au Québec en 1993 et en Ontario, selon Whitney (1988). Localisation des plantations de Bristol (1), de Buckingham (2), de Mayo 3, de Harrington (4, de Saint-Philippe-d'Argenteuil 5 au Québec et de la forêt Larose 6 en Ontario. 
maladie au Québec. L'opération de détection de la maladie du rond ainsi amorcée a permis de cumuler des observations sur une période de plus de 10 ans.

Afin d'être en mesure de détecter la maladie du rond, quatre plantations de pins rouges ont été sélectionnées. Cellesciavaient subi une éclaircie sans traitement préventif des souches. Situées dans la vallée de la rivière des Outaouais, en amont et en aval de la forêt Larose mais du côté du Québec (fig.1), ces quatre plantations sont : Bristol (comté de Pontiac; $45^{\circ} 32^{\prime} \mathrm{N}$ $76^{\circ} 30^{\prime} \mathrm{O}$ ), Buckingham (comté de Papineau; $\left.45^{\circ} 33^{\prime} \mathrm{N} 75^{\circ} 28^{\prime} \mathrm{O}\right)$; Mayo près du lac La Blanche (comté de Papineau; $45^{\circ} 44^{\prime} \mathrm{N} 75^{\circ} 20^{\prime}$ O) et Saint-Philipped'Argenteuil (comté d'Argenteuil; $45^{\circ} 36^{\prime} \mathrm{N}$ $\left.74^{\circ} 28^{\prime} \mathrm{O}\right)$. Ces plantations ont été éclaircies en 1981, sauf la plantation de Bristol qui a été éclaircie en 1983.

De 1983 à 1993, à chaque période automnale, nous avons procédé à des visites dans chacune des plantations à la recherche d'arbres morts ou dépérissants. Lorsque de tels arbres étaient localisés, leur base était dénudée de toute matière organique, à la recherche des fructifications du champignon. Les fructifications trouvées étaient apportées au laboratoire pour identification. Le champignon était isolé en culture pure et l'identification était basée sur la production de conidies caractéristiques de l'anamorphe de $\mathrm{H}$. annosum, le Spinigermeineckella(Olsom) Stalpers, tel que décrit par Phillips et Burdekin (1982).

À l'automne 1988, des pins morts ont été repérés dans la plantation de Mayo près du lac La Blanche. Cependant, ce n'est qu'en septembre 1989 que des fructifications de champignons ont été trouvées sur ces arbres. En laboratoire, ces champignons ont été formellement identifiés comme étant le $H$. annosum. Une culture ( $n^{\circ}$ 89-059) et des spécimens d'herbier (QFB 19023) ont été déposés dans les collections du Centre de foresterie des Laurentides (CFL) du Service canadien des forêts - Québec. L'identification du H. annosum dans cette plantation de pins rouges en 1989 constitue donc la première mention vérifiée de la maladie du rond au Québec (Laflamme et Blais 1993). La source présumée d'infection, soit la forêt
Larose, en Ontario, se trouve à environ 40 $\mathrm{km}$ de cet endroit.

En 1993, quelques arbres dépérissants ont été localisés à deux endroits dans une autre plantation, celle de Saint-Philipped'Argenteuil; ils étaient situés près de souches provenant de l'éclaircie de 1981. Les fructifications recueillies à ces endroits ont permis d'identifier formellement le $H$. annosum en laboratoire. Une culture ( $n^{\circ}$ 93-006) et des spécimens d'herbier (QFB 19072) ont également été déposés dans les collections du CFL. Les vérifications des plantations de Bristol et de Buckingham n'ont pas permis de détecter la maladie à ces deux endroits.

Le pin rouge est actuellement l'essence la plus vulnérable à la maladie du rond dans l'est du Canada. Toutefois, on a noté que quelques plantations de pins gris, de pins sylvestres et de pins blancs avaient également été atteintes par la maladie en Ontario (Sippell et al. 1967). Par contre, nous avons observé que la maladie semblait moins spécifique chez les semis et chez la régénération, et qu'elle pouvait causer la mort relativement rapidement sur des espèces autres que le pin rouge. Dans un centre d'infection de la plantation de Mayo, par exemple, la régénération naturelle a été très affectée, car on a noté de la mortalité chez des pins rouges de $2 \mathrm{~cm}$ diam au collet et chez quelques pins blancs d'environ $10 \mathrm{~cm}$ diam. De plus, des fructifications du $H$.annosum ont été retrouvées sur des sapins baumiers [Abies balsamea (L.) Mill.] en régénération et sur des cerisiers de Pennsylvanie (Prunus pensylvanica L.). Ces observations illustrent bien la problématique du reboisement sur des sites déjà infectés par le H. annosum.

Après avoir informé les forestiers de la présence de la maladie du rond au Québec (Laflamme et Blais 1990), et après avoir fait une description des symptômes de la maladie (Laflamme 1993), deux autres plantations montrant des ronds de mortalité ont été signalées, une à Berthierville $\left(46^{\circ} 08^{\prime} N 73^{\circ} 12^{\prime} \mathrm{O}\right)$ et l'autre à Harrington $\left(45^{\circ} 47^{\prime} \mathrm{N} 74^{\circ} 40^{\prime} \mathrm{O}\right)$. Après vérification, la mortalité dans la plantation de conifères mixtes, près de Berthierville, était causée par un insecte, le Dendroctonus valens Leconte [Coleoptera: Scolytidae]. Par contre, dans la plantation de pins rouges 
située près de Harrington, nous avons pu récolter, en 1993, de nombreuses fructifications et identifier le $\mathrm{H}$. annosum en culture pure. Des spécimens (QFB 19372) et une culture ( ${ }^{\circ}$ 93-007) ont été déposés dans les collections du CFL. Comme l'éclaircie a eu lieu en 1977 à Harrington, il est fort probable que l'infection a eu lieu la même année. À ce titre, cette plantation aurait donc été infectée avant celle de Mayo et serait, de fait, la première plantation infectée au Québec.

Dans la plantation de Mayo, l'éclaircie a eu lieu en 1981. Par contre, les arbres morts n'ont été détectés qu'en 1988 et les fructifications du champignon en 1989. La maladie progresse donc très lentement à partir du point d'infection initial. Comme les symptômes n'apparaissent pas avant 7 ou 8 ans après l'éclaircie, les relevés des maladies ne devraient pas se faire avant cette période de temps. Bien que la vitesse de propagation soit faible à partir d'une souche, le nombre de points d'infection est potentiellement très élevé lors d'une éclaircie dans une plantation. Par exemple, 12 centres d'infection ont été trouvés dans la plantation de Mayo en 1992. Par la suite, lorsque le $H$. annosum s'installe dans un peuplement, il est très difficile de l'éliminer car il peut y végéter pendant plusieurs décennies. Greig et Pratt (1976) ont observé que le $H$. annosum avait survécu dans de vieilles souches jusqu'à 62 ans, ce qui ne serait pas un maximum absolu. C'est cette caractéristique de permanence du champignon sur un site donné qui rend la maladie du rond si dévastatrice.

Au Québec, dans le cas des plantations infectées, les centres d'infection sont encore relativement petits et peu nombreux. II est donc encore possible d'éradiquer la maladie du rond en enlevant les souches infectées et en les détruisant. II faudrait aussi songer à éliminer le centre présumé d'infection de la forêt Larose. Enfin, pour empêcher la progression de la maladie au Québec, il faudrait traiter les souches de façon préventive soit avec du borax (Myren 1981), soit avec un produit biologique comme le champignon Peniophora gigantea (Fr.) Massee, lequel fut utilisé pour la première fois par Rishbeth (1963) en Angleterre, et ce, dès que ce produit sera commercialement disponible.

\section{REMERCIEMENTS}

Les auteurs remercient Gregory Crook, anciennement de la Compagnie internationale de papier du Canada (CIP), et Fernand Robichaud d'Avenor inc.; Guy Bussières, Martine Cormier, Geneviève Roy et André Dansereau de I'Université Laval; Louise Innes et le personnel du ministère des Ressources naturelles du Québec; et Pierre Duval du Service canadien des forêts - Québec (Ressources naturelles Canada).

\section{RÉFÉRENCES}

Dimitri, L. 1973. Root-rot caused by Fomes annosus in the forests of Western Europe : Frequency, damages and current research work. Eur. J. For. Pathol. 3: 245-248.

Greig, J.W. et J.E. Pratt. 1976. Some observations on the longevity of Fomes annosus in conifer stumps. Eur. J. For. Pathol. 6: 250-253.

Hord, H.H.V. et D.A. Quirke. 1955. Province of Ontario: Forest disease survey. Pages 56-69 in Annual report of the forest insect and disease survey. Dep. Agric., Ottawa, Ontario.

Laflamme, G. 1993. La maladie du rond Ordre Ing. For. Qué. L'Aubelle n ${ }^{\circ 5}$, Avril.

Laflamme, G. et R. Blais. 1990. Maladie du rond. Page 32 in Insectes et maladies des arbres: Québec 1989. For. Conserv. 57 (supplément).

Laflamme, G. et R. Blais. 1993. Première mention de Heterobasidion annosum au Québec. Phytoprotection 74: 171.

Mains, E.B., L.O. Overholts et R. Pomerleau. 1939. Fungi collected at the Foray, August 1938. Mycologia 31: 728-736.

Myren, D.T. 1981. Use of Borax and sodium nitrite in an operational thinning of red pine in Ontario to prevent stump infection by Fomes annosus. For. Chron. $57: 284-$ 285.

Phillips, D.H. et D.A. Burdekin. 1982. Diseases of forest and ornamental trees. MacMillan Press, London. $435 \mathrm{pp}$.

Redfern, D.B. et G.A. Van Sickle. 1968. Fomes annosus in eastern Canada. Plant Dis. Rep. 52: 638. 
Rishbeth, J. 1951. Observations on the biology of Fomes annosus with particular reference to East Anglia pine plantations. II. Spore production, stump infection, and saprophytic activity in stumps. Ann. Bot. (Londres) 15: 1-21.

Rishbeth, J. 1959. Dispersal of Fomes annosus and Peniophora gigantea. Trans. Br. Mycol. Soc. 42 : 243-260.

Rishbeth, J. 1963. Stump protection against Fomes annosus. III. Inoculations with Peniophora gigantea. Ann. Appl. Biol. 52: 63-77.
Sippell, W.L., A.H. Rose et M.J. Larsen. 1967. Ontario Region. Pages 69-70 in Annual report of the forest insect and disease survey - 1967. Dep. For. and Rural Develop., Ottawa, Ontario.

Sippell, W.L., H.L. Gross et A.H. Rose. 1968. Ontario Region. Page 72 in Annual report of the forest insect and disease survey 1968. Dep. Fish. For., Ottawa, Ontario.

Whitney, R.D. 1988. L'ennemi caché. Transfert de la technologie concernant les pourridiés. Min. Rich. nat. Ont. et Serv. can. for., Sault Ste. Marie, Ontario. $35 \mathrm{pp}$. 